\title{
Mode-locked laser pulse trains with subfemtosecond timing jitter synchronized to an optical reference oscillator
}

\author{
A. Bartels, S. A. Diddams, T. M. Ramond, and L. Hollberg \\ Time and Frequency Division, National Institute of Standards and Technology, 325 Broadway, Boulder, Colorado 80305
}

\begin{abstract}
We independently phase lock the repetition rates of two femtosecond lasers at their $\approx 456,000$ th harmonic to a common optical oscillator. The timing jitter of each individual laser relative to the optical reference is only $0.45 \mathrm{fs}$ in a $100-\mathrm{Hz}$ bandwidth. Our method takes advantage of the tremendous leverage that is possible when stability is transferred from the optical to the microwave domain. The low timing jitter is commensurate with the independently measured fractional frequency instability in the repetition rates of $\leq 2.3 \times 10^{-15}$ in 1 -s averaging time, limited by the measurement system. The microwave signals at $1 \mathrm{GHz}$ that are extracted by photodetection of the pulse trains have a 10-times-greater instability, confirming the presence of excess noise in the photodetection.
\end{abstract}

Mode-locked femtosecond lasers have proved to be powerful tools in the field of optical frequency metrology, where they provide a convenient phasecoherent link between the microwave and optical domains. ${ }^{1-3}$ Research has rapidly progressed from the direct measurement of optical frequencies and frequency ratios ${ }^{4}$ to the creation of clocks referenced to optical transitions in atoms, molecules, or single trapped ions., ${ }^{5,6}$ The most immediate benefit of these new clocks based on optical transitions is their much improved stability. Indeed, the stability of atomic frequency references with carrier frequency $\nu_{0}$ and linewidth $\Delta \nu$ scales with $Q=\nu_{0} / \Delta \nu$. It is thus probable that in the near future the ultimate stability performance will belong to laser oscillators referenced to narrow atomic transitions. With this in mind, we suggest that impor tant applications (such as radar, electro-optic sampling, optical analog-to-digital conversion, and precise timing) that currently rely on stable, low-phase-noise microwave oscillators and synchronization techniques may ultimately benefit from a low-noise optical oscillator that has its frequency downconverted to the microwave domain with a mode-locked femtosecond laser.

In this Letter we synchronize two femtosecond lasers at $1-\mathrm{GHz}$ repetition rate to a common stabilized optical reference oscillator at $\approx 456 \mathrm{THz}$. Our ability to transfer stability from the optical to the microwave domain is tested by comparison of the emerging pulse trains. Phase locking the repetition rates at their $\approx 456,000$ th harmonic allows us to achieve subfemtosecond timing jitter relative to the optical frequency reference. Our experiment can be viewed as an extension of recent results reported by Shelton et al., ${ }^{7}$ who phase locked two femtosecond lasers to each other at a harmonic of the repetition rates in the microwave domain. They achieved subfemtosecond timing jitter between the lasers in an experiment in which one laser was tracking the free-r unning repetition rate of the other. Aside from the ability to exploit the high stability of optical frequency references for the generation of highly stable microwave pulse trains, our method also allows us to circumvent noise-floor limitations in microwave components that are present when one is creating phase locks at low-order harmonics of the repetition rates. We have confirmed the high stability in the frequency domain: The fractional frequency instability of the repetition rates, characterized with the Allan deviation, ${ }^{8}$ is found to be $\leq 2.3 \times 10^{15}$ in 1 -s averaging time, limited by the measurement system.

The two femtosecond lasers under comparison ${ }^{9,10}$ as well as the methods for their stabilization ${ }^{2,11}$ have been described in detail elsewhere. The first laser is a ring oscillator at a repetition rate of $f_{r, 1} \approx 1 \mathrm{GHz}$. It is spectrally broadened in a microstructure fiber ${ }^{12}$ to span more than one octave and thus allow a measurement of its carrier-envelope offset frequency, $f_{0,1}$. The second laser directly emits a broadband continuum, thereby avoiding the need for an external broadening step to measure its offset frequency, $f_{0,2}$. Its repetition rate, $f_{r, 2}$, is close or equal to $f_{r, 1}$. Both lasers are pumped by individual solid-state pump lasers. The offset frequencies of both lasers are determined in a self-referencing technique and independently phase locked relative to a hydrogen maser by control of the pump power for each laser. Subsequently, we heterodyne a single-frequency laser diode $\left(f_{\mathrm{LD}} \approx 456 \mathrm{THz}\right)$ that is stabilized to a temperature-controlled Fabry-Perot cavity with the neighboring components of both frequency combs (with mode numbers $n_{1}$ and $n_{2}$, both approximately 456,000 ) to generate beat signals at frequencies $f_{b, 1}=f_{\mathrm{LD}} \quad f_{n 1}$ and $f_{b, 2}=f_{\mathrm{LD}} \quad f_{n 2}$. These beat signals are phase locked by control of the cavity length of each laser. The repetition rates of both lasers are thus phase locked to $f_{\mathrm{LD}}$ as $f_{r, i}=n_{i}{ }^{1} \times\left(\begin{array}{lll}f_{\mathrm{LD}} & f_{0, i} & f_{b, i}\end{array}\right)$ with $i=1,2$.

The quality of the stabilized optical pulse trains is evaluated with the basic apparatus shown in Fig. 1. We first employ an optical cross-correlation technique, using type I sum frequency generation in a lithium iodate crystal of $0.5-\mathrm{mm}$ thickness. Approximately $10 \mathrm{~mW}$ of output of each laser is focused into the nonlinear crystal to spatially overlap in a noncollinear configuration. The background-free cross-correlation signal is detected with a photomultiplier tube. To characterize the phase 


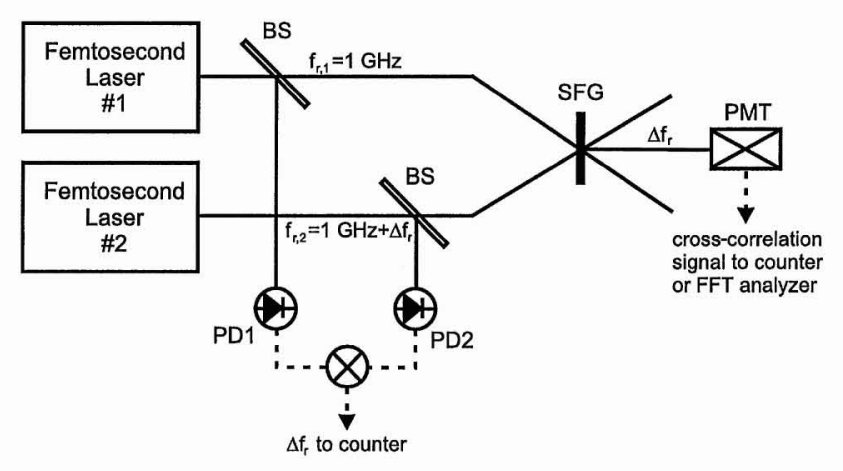

Fig. 1. The pulse trains pass through the beam splitters (BS) and generate a cross-correlation signal based on sum frequency generation in a lithium iodate crystal that is detected by the photomultiplier (PMT). This signal is either used as a probe for the relative phase of the pulse trains in the case of equal $f_{r, i}$ or delivers $\Delta f_{r}$ when the repetition rates are different. The portions that are reflected by the BSs are photodetected by photodiodes PD1 and PD2. Subsequently $\Delta f_{r}$ is electronically generated by a frequency mixer. FFT, fast Fourier transform.

noise, we set $f_{r, i}$ to exactly equal values by choosing $n_{1}=n_{2}$ and $f_{0,1}+f_{b, 1}=f_{0,2}+f_{b, 2}$. Subsequently, the time delay, $\Delta t$, between the pulse trains is chosen so that the cross-correlation signal $|U(\Delta t)|$ has a static nonzero value on a linear slope (point A in the inset of Fig. 2). Thus $U(\Delta t)$ is a probe for the time delay between the pulse trains. The slope $\mathrm{d} U / \mathrm{d} \Delta t$ has been extracted from the cross-correlation trace at point A. The convoluted timing noise spectrum of the two pulse trains, $S_{\Delta t}(f)$, is then obtained from an amplitude-noise power spectral density measurement $S_{U}(f)$ of $U(\Delta t)$ performed with a fast Fourier transform analyzer as $S_{\Delta t}(f)=(\mathrm{d} U / \mathrm{d} \Delta t){ }^{2} S_{U}{ }^{2}(f)$. The phase-noise spectral density is $L(f)=4 \pi^{2} f_{r, i}{ }^{2} S_{\Delta t}(f)$. Both results are displayed in Fig. 2 as a single curve with the appropriate scale. $L(f)$ is approximately $30-50 \mathrm{~dB}$ better than what is attainable with high-quality synthesizers and quartz oscillators and is comparable to results obtained with the best sapphire microwave oscillators. The pronounced excess-noise contribution in the range $0.1-1 \mathrm{kHz}$ is attributed to mechanical vibrations of the setup. We extract the timing jitter in a frequency range from $1 \mathrm{~Hz}$ to $B$ as $\sigma_{\Delta t}(B)=\left[\int_{1 \mathrm{~Hz}}^{B} S_{\Delta t}(f) \mathrm{d} f\right]^{1 / 2}$. In a passband of 1 to $100 \mathrm{~Hz}$, the timing jitter is as low as $0.45 \mathrm{fs}$; at our full available frequency span from 1 to $100 \mathrm{kHz}$, it increases to $1.5 \mathrm{fs}$. By repeating the experiment at point $\mathrm{B}$ of the cross-correlation trace, we have confirmed that amplitude noise contributes a negligible amount to our phase-noise measurement. Our two femtosecond lasers are of very different design, spatially separated and pumped by different pump lasers; no specific efforts toward obtaining a vibration-isolated setup have been made. Considering this, it is worth noting that we achieved a timing jitter comparable with what was achieved by Shelton et al. with elaborate vibration isolation. ${ }^{7}$ We attribute this result to the fact that phase locking an optical harmonic of the repetition rate to an optical reference divides the residual errors of our phase-locked loops by a factor of $\approx 456,000$ instead of the low factor that can be achieved with microwave-domain phase locking.

To further evaluate the transfer of frequency stability from the optical domain to $1-\mathrm{GHz}$ repetition rates, we set $f_{r, i}$ to have a difference of $\Delta f_{r} \approx 10 \mathrm{kHz}$. We generate $\Delta f_{r}$ with the two different methods described below and count it with a frequency counter at different gate times $\tau$. As a measure of fractional frequency uncertainty we then calculate the Allan deviation, $\sigma_{\Delta f_{r}}(\tau)$, of a number of readings. Assuming that fluctuations in both repetition rates contribute equally to fluctuations of $\Delta f_{r}$, we extract the Allan deviation ${ }^{8}$ of the repetition rate of each laser to $\sigma_{f_{r, i}}(\tau)=\sigma_{\Delta f_{r}}(\tau) \times \Delta f_{r} /\left(\sqrt{2} \times f_{r, i}\right)$. Here, it is important to point out that, because $f_{r, i}$ are locked to a common mode, $\sigma_{f, i}$ reflect the instability introduced by the mode-locked lasers themselves, as if the lasers were locked to an ideally stable reference. Our experiment is therefore a rigorous test of the stability transfer properties from the optical to the microwave domain of the femtosecond lasers. The frequency counter imposes a resolution limit of $\approx 2 \times 10^{15} \tau^{1}$ on our capability to measure $\sigma_{f r, i}(\tau)$. The phase-locked loops create an error in $f_{r, i}$ that is negligible compared with the frequency-counter limit.

For a direct measurement of the repetition rate stability, $\Delta f_{r}$ can be immediately derived from the nonlinear cross-correlation signal obtained as described above. The photomultiplier's output is amplified and filtered with a $30-\mathrm{kHz}$ low-pass filter and enters a frequency counter to yield readings of $\Delta f_{r}$. The corresponding Allan deviations $\sigma_{f_{r, i}}(\tau)$ of the measurements at various gate times $\tau$ are displayed as squares in Fig. 3. The error bars represent a statistical uncertainty of $N^{1 / 2}$, where $N$ is the number of sample points at the respective gate times. At 1-s gate time the instability of the repetition rates is $\leq 2.3 \times 10^{15}$. For longer gate times it averages down as $\tau^{1}$, limited by the resolution of the counter. The value of $\sigma_{f_{r, i}}$ $(100 \mathrm{~ms})$ is $7.6 \times 10^{14}$, deviating from the $\tau^{1}$ behavior. Acoustic modulations of the different path lengths

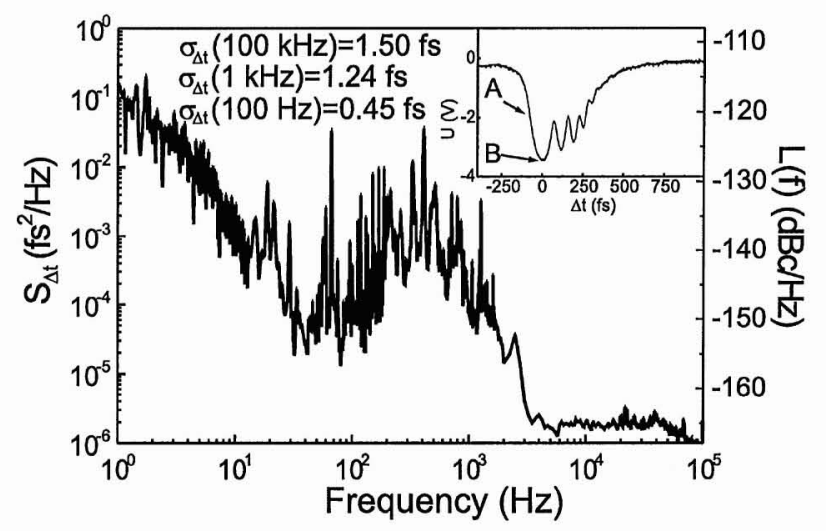

Fig. 2. Timing-noise (left-hand axis) and phase-noise (right-hand axis) spectrum of the two laser pulse trains. Their timing jitter is given for different bandwidths. The inset shows a cross-correlation signal between the two pulse trains. Its broad structured shape stems from the fact that one of the pulse trains passed a number of optical components before reaching the cross-correlation crystal. 


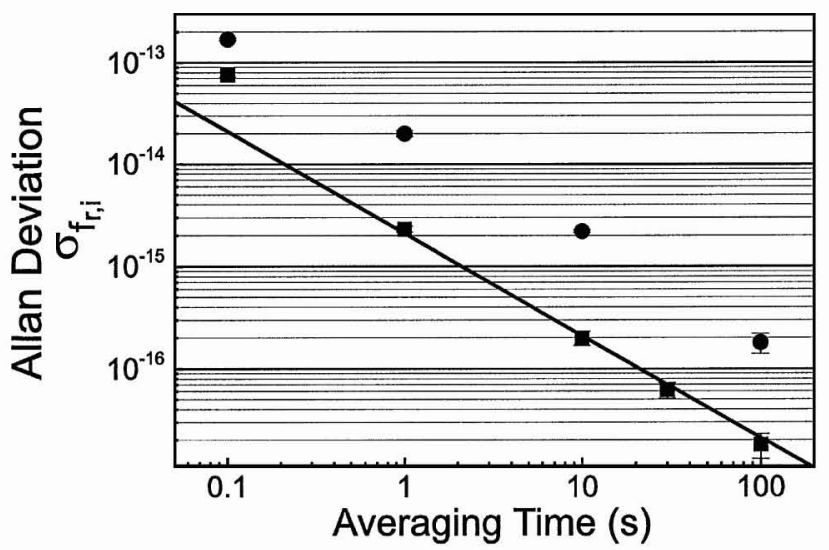

Fig. 3. $\sigma_{f_{r, i}}$ (see text for definition) of the two lasers as measured by mixing of the photodetected electronic signals (circles) and with the cross-correlation technique (squares). The solid line represents the measurement limit given by our frequency counter.

of the two pulse trains that traveled to the nonlinear crystal may be responsible for this instability at short time scales.

To test our ability to extract stable electronic signals, we individually photodetect the pulse trains at $1 \mathrm{GHz}$ with two silicon p-i-n photodiodes. Approximately $5 \mathrm{~mW}$ of power are incident on each diode and generate $\sim 10 \mathrm{dBm}$ of microwave power in the $1-\mathrm{GHz}$ harmonic, with a signal-to-noise ratio of better than $95 \mathrm{~dB}$ in a $300-\mathrm{kHz}$ bandwidth. The amplified signals are supplied to a frequency mixer to again generate a $\Delta f_{r}$ signal (see Fig. 1), which is filtered with a $30-\mathrm{kHz}$ low-pass filter before it enters the frequency counter. The Allan deviations of the $1-\mathrm{GHz}$ electronic signals counted at various gate times are displayed as circles in Fig. 3. At a 1-s gate time, the $\sigma_{f_{r, i}}(\tau)$ are $2 \times 10^{14}$. The $\sigma_{f_{r, i}}(\tau)$ exhibit the same $\tau^{1}$ behavior as the values obtained with the cross-correlation technique, but are almost 1 order of magnitude larger than those of the optical pulse trains. We have estimated the detector shot-noise limit to the stability ${ }^{13}$ of our microwaves and found that it should be below $1 \times 10^{15}$ for our configuration. Thus our result shows that the photodetection process or the subsequent electronic processing corrupts the very stable optical pulse trains. Independent tests of the electronic components indicate that the dominant source of noise arises in the photodetectors themselves. Ivanov et al. identified amplitude-to-phase noise conversion and pointing instability to be two source of excess noise. ${ }^{14}$ However, spatial filtering in optical fibers and active stabilization of the optical power reaching the detectors still have not yet allowed us to reproduce the proven stability of the optical pulse trains in the electronic microwave signals. The presence of this excess noise in photodetection and the subsequent electronics has important implications for many optoelectronic applications, including the generation of very stable electronic clock signals.

In conclusion, we have demonstrated the synchronization of two independent laser pulse trains to an optical reference oscillator with 450 -as relative timing jitter in a $100-\mathrm{Hz}$ bandwidth and with a relative fractional frequency instability of $\leq 2.3 \times 10^{15}$ in 1 -s averaging time. With no specific efforts toward vibrational damping, these results are made possible by the great leverage on phase noise that is possible by transfer of stability from the optical to the microwave domain. Excess noise in the photodetectors still corrupts the stability of the optical pulse trains when they are converted to a microwave signal. However, with a fractional instability of $2 \times 10^{14} \tau^{1}$ we are able to create a microwave signal with very high stability that is referenced to an optical oscillator, surpassing the performance of current microwave standards. Our microwave synthesis technique should ultimately allow us to take full advantage of the ultralow phase noise and instability of optical frequency standards approaching $10^{16}$ in $1 \mathrm{~s}^{15}$

We are grateful to C. W. Oates, E. A. Curtis, and E. Ivanov for their contributions to these experiments and to R. Windeler of OFS for generously providing the microstructure fiber used in one of the lasers. A. Bartels's e-mail address is albrecht@boulder.nist.gov.

\section{References}

1. Th. Udem, J. Reichert, R. Holzwarth, and T. W. Hänsch, Phys. Rev. Lett. 82, 3568 (1999).

2. S. A. Diddams, D. J. Jones, J. Ye, S. T. Cundiff, J. L. Hall, J. K. Ranka, R. S. Windeler, R. Holzwarth, T. Udem, and T. W. Hänsch, Phys. Rev. Lett. 84, 5102 (2000).

3. R. Holzwarth, Th. Udem, T. W. Hänsch, J. C. Knight, W. J. Wadsworth, and P. St. J. Russell, Phys. Rev. Lett. 85, 2264 (2000).

4. J. Stenger, H. Schantz, Ch. Tamm, and H. R. Telle, Phys. Rev. Lett. 88, 073601-1 (2002).

5. S. A. Diddams, Th. Udem, J. C. Bergquist, E. A. Curtis, R. E. Drullinger, L. Hollberg, W. M. Itano, W. D. Lee, C. W. Oates, K. R. Vogel, and D. J. Wineland, Science 293, 825 (2001).

6. J. Ye, L.-S. Ma, and J. L. Hall, Phys. Rev. Lett. 87, 270801 (2001).

7. R. K. Shelton, S. M. Foreman, L.-S. Ma, J. L. Hall, H. C. Kapteyn, M. M. Murnane, M. Notcutt, and J. Ye, Opt. Lett. 27, 312 (2002).

8. D. W. Allan, IEEE Trans. Ultrason. Ferroelectr. Freq. Control 34, 647 (1987).

9. A. Bartels, T. Dekorsy, and H. Kurz, Opt. Lett. 24, 996 (1999).

10. A. Bartels and H. Kurz, Opt. Lett. 27, 1839 (2002).

11. T. M. Ramond, S. A. Diddams, L. Hollberg, and A. Bartels, Opt. Lett. 27, 1842 (2002).

12. J. K. Ranka, R. S. Windeler, and A. J. Stenz, Opt. Lett. 25, 25 (2000).

13. L. Hollberg, C. W. Oates, E. A. Curtis, E. N. Ivanov, S. A. Diddams, Th. Udem, H. G. Robinson, J. C. Bergquist, R. J. Rafac, W. M. Itano, R. E. Drullinger, and D. J. Wineland, IEEE J. Quantum Electron. 37, 1502 (2001).

14. E. N. Ivanov, L. Hollberg, and S. A. Diddams, in Proceedings of the 2002 IEEE International Frequency Control Symposium (Institute of Electrical and Electronics Engineers, Piscataway, N.J., 2002).

15. B. C. Young, F. C. Cruz, W. M. Itano, and J. C. Bergquist, Phys. Rev. Lett. 82, 3799 (1999). 